\title{
MORE ON FINITE GROUPS WITH FEW DEFINING RELATIONS
}

\author{
J. L. MENNICKE and B. H. NEUMANN
}

(Received 8 April 1987)

Communicated by H. Lausch

\begin{abstract}
Certain central products of the binary polyhedral groups with finite cyclic groups are here shown to have presentations with two generators and two defining relations; this disproves a conjecture of the second author, stated in J. Austral. Math. Soc. Ser. A 38 (1985), 230-240.
\end{abstract}

1980 Mathematics subject classification (Amer. Math. Soc.) (1985 Revision): 20 F 05.

\section{Introduction}

The first author has destroyed a conjecture of the second author: we here present the history and the result.

In [3] it had been shown that central products of binary polyhedral groups and cyclic groups of (finite) even order have presentations with 3 generators and 3 defining relations, so-called "zero-deficiency" or "balanced" presentations. The groups in question can also be generated by two of the generators, but the second author did not know whether they could be defined by two relations in those generators; and he stuck out his neck by conjecturing [3, page 239] that there are many cases when they could not.

The second author gratefully acknowledges financial support from the DAAD (Deutscher Akademischer Austauschsdienst) and the generous hospitality of the Fakultät für Mathematik der Universität Bielefeld while this paper was written.

(C) 1989 Australian Mathematical Society 0263-6115/89 \$A2.00+0.00 
In the present note we show that all these groups can be defined by two relations in those two generators; thus the conjecture of the second author is false.

The first author had, in fact, already dealt with some of the cases in his papers [1], [2], based on methods and results of Threlfall and Seifert [4], [5]; however, the aim and flavour of these papers is topological, and the fact that they yield balanced presentations of the relevant groups is not obvious to a mere group theorist.

\section{The presentations}

In [3], the presentations of groups $G(p, q, r, s, t, u)$ are considered; the generators are $a, b, c$, and the relations are

$$
\left\{\begin{array}{l}
a^{p}=c^{s}, \\
b^{q}=c^{t}, \\
(a b)^{r}=c^{u} .
\end{array}\right.
$$

The parameters $p, q, r, s, t, u$ are integers subject to the conditions

$$
\left\{\begin{array}{l}
0<p \leq q \leq r, \\
\frac{1}{p}+\frac{1}{q}+\frac{1}{r}>1, \\
(s, t)=(s, u)=(t, u)=1, \\
\Delta:=-p q u+p r t+q r s \neq 0 .
\end{array}\right.
$$

It is then shown that the groups thus defined are finite. If $p=1$, the groups are cyclic, and we dismiss this case as of no interest here. When $p=2$ (greater values do not occur, because of the conditions (B)), the groups are extensions of a cyclic central subgroup $C_{2 n}$ of even order $2 n$ by a polyhedral group. The polyhedral groups are

the dihedral groups $D_{2 r}$ of order $2 r$ when $p=q=2, r \geq 2$ arbitrary;

the tetrahedral group $T_{12}$ of order 12 when $p=2, q=r=3$;

the octahedral group $O_{24}$ of order 24 when $p=2, q=3, r=4$;

the icosahedral group $I_{60}$ of order 60 when $p=2, q=3, r=5$.

The order $2 n$ of the cyclic group can be made equal to an arbitrarily prescribed positive even number, by suitable choice of the remaining parameters $s, t, u$. 
Details can be found in [3]. We just summarise the results for $n$ :

in the dihedral case with odd $r$,

$$
n=r s+r t-2 u
$$

in the dihedral case with even $r$,

$$
n=\frac{1}{2} r s+\frac{1}{2} r t-u
$$

in the tetrahedral case,

$$
n=3 s+2 t-2 u \text {; }
$$

in the octahedral case,

$$
n=6 s+4 t-3 u
$$

in the icosahedral case,

$$
n=15 s+10 t-6 u \text {. }
$$

As the values of the parameters are of no great interest here, we shall use briefly $G(P, n)$, where $P$ is one of the polyhedral groups, for the $G(p, q, r, s, t, u)$ which is the extension of a central $C_{2 n}$ by $P$.

It is shown in $[3$, page 329] that the groups $G(P, n)$ can, by virtue of the conditions (B) we have imposed, be generated by $a$ and $b$ alone. We now show that they can be defined by two relations in these two generators. The dihedral case is slightly different from the other polyhedral cases, so we treat it separately. It divides into several cases according to the parity of $r$ and that of $n$ and, when both are odd, also the parity of half their sum.

(a) Let $r$ and $n$ be both odd. As

$$
n=r s+r t-2 u \text {, }
$$

we must ensure that $r(s+t)$ is odd; this we do by choosing $s=1$ and $t=2$ or $t=4$ and $u=\frac{1}{2}(-n+3 r)$ or $\frac{1}{2}(-n+5 r)$, whichever of these two is odd; this ensures that $(t, u)=1$. The choice of $s=1$ ensures that also $(s, t)=(s, u)=1$, and it means that $a^{2}=c$, so $c$ can be replaced by $a^{2}$ and then omitted as a generator. Thus we get the presentations

$$
G\left(D_{2 r}, n\right) \cong g p\left(a, b ; b^{2}=a^{4},(a b)^{r}=a^{-n+3 r}\right)
$$

or

$$
G\left(D_{2 r}, n\right) \cong g p\left(a, b ; b^{2}=a^{8},(a b)^{r}=a^{-n+5 r}\right),
$$

according to whether $n \equiv r$ or $-r(\bmod 4)$.

(b) Let $r$ be odd, but $n$ even. Still

$$
n=r s+r t-2 u
$$


but now we can put $s=t=1$ and $u=-\frac{1}{2} n+r$ and get the presentation

$$
G\left(D_{2 r}, n\right) \cong g p\left(a, b ; a^{2}=b^{2},(a b)^{r}=a^{-n+2 r}\right) .
$$

(c) Let $r$ be even. Now we have

$$
n=\frac{1}{2} r s+\frac{1}{2} r t-u,
$$

so we can put $s=t=1, u=-n+r$, and get the presentation

$$
G\left(D_{2 r}, n\right) \cong g p\left(a, b ; a^{2}=b^{2},(a b)^{r}=a^{-2 n-2 r}\right) .
$$

It is clear that we have chosen the parameters $s, t, u$ so that

$$
(s, t)=(s, u)=(t, u)=1
$$

-in fact it was this aim that made it necessary to consider so many cases. The result is, as claimed, that $G\left(D_{2 r}, n\right)$ can be defined by two relations in the generators $a$ and $b$. A simpler presentation might be possible if different generators are permitted, but our point is that a balanced presentation exists in the two given generators.

The other polyhedral cases are simpler, and we deal with them jointly. Consider the presentation

$$
g p\left(a, b ; a^{2}=\left(a^{2}(a b)^{r}\right)^{-s}, b^{3}=\left(a^{2}(a b)^{r}\right)^{-t}\right),
$$

where $r=3,4,5$ in the tetrahedral, octahedral, icosahedral case, respectively, and $s$ and $t$ are functions of $n$ to be described later. We put $u=-s-1$, and thus ensure $(s, u)=1$. Now if we further put $c=(a b)^{-r} a^{-2}$, then the relations become

$$
a^{2}=c^{s}, \quad b^{3}=c^{t} ;
$$

and it follows that $c=(a b)^{-r} c^{-s}$, and thus

$$
(a b)^{r}=c^{-s-1}=c^{u} .
$$

Thus we have recovered the defining relations (A). It only remains to fix $s, t$ appropriately. This is done separately for the three values of $r$.

When $r=3$, that is in the tetrahedral case, we have

$$
n=3 s+2 t-2 u,
$$

and put $s=n, t=-2 n-1, u=-n-1$. These are easily seen to be pairwise coprime, and to satisfy (3). Thus

$$
G\left(T_{12}, n\right) \cong g p\left(a, b ; a^{2}=\left(a^{2}(a b)^{3}\right)^{-n}, b^{3}=\left(a^{2}(a b)^{3}\right)^{2 n+1}\right)
$$

is a balanced presentation as required. 
When $r=4$, that is in the octahedral case, we have

$$
n=6 s+4 t-3 u,
$$

and put $s=n+1, t=-2 n-3, u=-n-2$. Again these are pairwise coprime and satisfy (4). Thus

$$
G\left(O_{24}, n\right) \cong g p\left(a, b ; a^{2}=\left(a^{2}(a b)^{4}\right)^{-n-1}, b^{3}=\left(a^{2}(a b)^{4}\right)^{2 n+2}\right)
$$

is a balanced presentation as required.

Finally, when $n=5$, that is in the icosahedral case, we have

$$
n=15 s+10 t-6 u,
$$

and put $s=n+4, t=-2 n-9, u=-n-5$. As before these are pairwise coprime and satisfy (5). Thus

$$
G\left(I_{60}, n\right) \cong g p\left(a, b ; a^{2}=\left(a^{2}(a b)^{5}\right)^{-n-4}, b^{3}=\left(a^{2}(a b)^{5}\right)^{2 n+9}\right)
$$

is a balanced presentation as required. This verifies our claim that all $G(P, n)$ have a presentation with only two defining relations in $a$ and $b$.

\section{References}

[1] J. Mennicke, 'Über Heegarddiagramme vom Geschlecht zwei mit endlicher Fundamentalgruppe', Arch. Math. 8 (1957), 192-198.

[2] J. Mennicke, 'Über endliche Fundamentalgruppen', Unpublished manuscript (1956).

[3] B. H. Neumann, 'Some finite groups with few defining relations', J. Austral. Math. Soc. Ser. A 38 (1985), 230-240.

[4] W. Threlfall and H. Seifert, 'Topologische Untersuchung der Diskontinuitätsbereiche endlicher Bewegungsgruppen des dreidimensionalen sphärischen Raumes', Math. Ann. 104 (1931), 1-70.

[5] W. Threlfall and H. Seifert, 'Topologische Untersuchung der Diskontinuitätsbereiche endlicher Bewegungsgruppen des dreidimensionalen sphärischen Raumes (SchluB)', Math. Ann. 107 (1933), 543-586.

Fakultät für Mathematik

Universität Bielefeld

D-4800 Bielefeld 1

Germany
Department of Mathematics Institute of Advanced Studies Australian National University Canberra, ACT 2601 and

Division of Mathematics and Statistics Commonwealth Scientific and Industrial Research Organization Canberra, ACT 2601 Australia 